\title{
Leiomiosarcoma primario de hueso en fémur distal metastásico a clavícula. Reporte de un caso
}

\section{Primary Leiomyosarcoma of Bone in the Distal Femur with Clavicle Metastasis. A Case Report}

\author{
1 Especialista en Cirugía Ortopédica Oncológica, Hospital \\ Universitario San Ignacio, Bogotá, Colombia \\ 2 Residente del IV año de Ortopedia y Traumatología, Hospital \\ Universitario San Ignacio, Bogotá, Colombia \\ ${ }^{3}$ Estudiante de XII semestre, Unidad de Ortopedia, Hospital \\ Universitario San Ignacio, Bogotá, Colombia
}

Francisco Linares R. ${ }^{1}$ Helbert Martín V. ${ }^{2}$ Natalia Correal A. ${ }^{3}$

Rev Chil Ortop Traumatol 2021;62(3):e221-e226.

\begin{abstract}
Dirección para correspondencia Helbert Martin V, Departamento de Ortopedia, Hospital Universitario San Ignacio, Carrera 7\#40-62, Bogotá, Colombia (e-mail: hmartin@husi.org.co).
\end{abstract}

\begin{abstract}
Resumen
Palabras Clave

- tumor óseo

- leiomiosarcoma

- metástasis ósea

El leiomiosarcoma (LMS) es un tipo de tumor de células fusiformes de muy baja incidencia, que tiene un comportamiento agresivo, con alta tasa de mortalidad, por lo que el manejo debe ser quirúrgico, con una resección amplia de la lesión. No está claro el papel de la radio ni de la quimioterapia en su manejo. Presentamos a una paciente de 28 años que consultó por dolor de 2 meses en la rodilla derecha. Radiográficamente, se caracterizó como una lesión osteolítica pura en el fémur distal. La resonancia nuclear magnética (RNM) contrastada mostró áreas hipervasculares dentro del tumor. La gammagrafía mostró un marcado aumento en la captación de radiotrazadores. Se tomó una biopsia, con un informe de patología de LMS óseo bien diferenciado. Se trató con 3 ciclos de quimioterapia neoadyuvante preoperatoria con ifosfamida $1.000 \mathrm{mg} / \mathrm{m}^{2}$ en los días 1 a 3, además de doxorrubicina $70 \mathrm{mg} / \mathrm{m}^{2}$, y resección quirúrgica de la lesión y salvamento de la extremidad con endoprótesis de rodilla. Una vez que se resecó la lesión, la paciente recibió quimioterapia adyuvante con 4 ciclos de gencitabina $1.000 \mathrm{mg} / \mathrm{m}^{2}$ entre los días 1 y 8 , y doxetacel $70 \mathrm{mg} / \mathrm{m}^{2}$ el día 1 . Durante los dos meses de seguimiento, la paciente presenóa una fractura en el tercio medio de la clavícula, compatible con una lesión patológica en radiografías y tomografía por emisión de positrones (TEP). La biopsia reveló una lesión metastásica de LMS óseo que fue tratada mediante resección quirúrgica de la clavícula. Este es un caso único, dado que, durante el seguimiento, recibió tratamiento adyuvante con quimioterapia y se evaluó con una TEP, con una evolución clínica satisfactoria y sin evidencia de nuevas lesiones.

Nivel de evidencia IV.
\end{abstract}

recibido

14 de mayo de 2020

aceptado

21 de enero de 2021
DOI https://doi.org/

10.1055/s-0041-1740233. ISSN $0716-4548$.
(C) 2021. Sociedad Chilena de Ortopedia y Traumatologia. All rights reserved.

This is an open access article published by Thieme under the terms of the Creative Commons Attribution-NonDerivative-NonCommercial-License, permitting copying and reproduction so long as the original work is given appropriate credit. Contents may not be used for commercial purposes, or adapted, remixed, transformed or built upon. (https://creativecommons.org/ licenses/by-nc-nd/4.0/)

Thieme Revinter Publicações Ltda., Rua do Matoso 170, Rio de Janeiro, RJ, CEP 20270-135, Brazil 


\begin{abstract}
Leiomyosarcoma (LMS) is a type of spindle-cell tumor of very low incidence that tumor has an aggressive behavior, with high mortality rates; therefore, its management must be surgical, with a wide resection of the lesion. The role of radio and chemotherapy in its management is not clear. We present the case of a 28 -year-old female patient who consulted for pain lasting 2 months in the right knee. Radiographically, it was characterized as a pure osteolytic lesion in the distal femur. Contrast magnetic resonance imaging (MRI) showed hypervascular areas within the tumor. The scintigraphy showed a marked increase in radiotracer uptake. A biopsy was taken, with a pathology report of well-differentiated osseous LMS. It was treated with 3 cycles of preoperative neoadjuvant chemotherapy with ifosfamide $1,000 \mathrm{mg} / \mathrm{m}^{2}$ in the first 3 days, as well as doxorubicin $70 \mathrm{mg} / \mathrm{m}^{2}$, and surgical resection of the lesion and limb salvage with knee endoprosthesis. Once the lesion was resected, the patient underwent adjuvant chemotherapy, with 4 cycles of gencitabine $1,000 \mathrm{mg} / \mathrm{m}^{2}$ between days 1 and 8 , and doxetacel $70 \mathrm{mg} / \mathrm{m}^{2}$ on day 1 . During the 2 -month follow-up, the patient presented a fracture in the middle third of the clavicle, which was compatible with a pathological lesion on radiographs and positron-emission tomography (PET) scans. The biopsy showed a metastatic lesion of bone LMS, which was treated by surgical resection of the clavicle. This is a unique case, given that, during

Keywords

- bone tumor

- leiomyosarcoma

- bone metastasis the follow-up, the patient underwent adjuvant treatment with chemotherapy, and was evaluated with a PET scan, with a satisfactory clinical evolution and no evidence of new lesions.

Level of evidence IV.
\end{abstract}

\section{Introducción}

El leiomiosarcoma (LMS) es un tipo de tumor de células fusiformes caracterizado por contener marcadores para músculo liso; no produce matriz osteoide, y su comportamiento es de tumor maligno. En general, se presenta en el retroperitoneo y el tracto gastrointestinal. ${ }^{1-3}$ Su presentación ósea es rara; en la literatura, la información respecto al LMS intraóseo es escasa, y la mayoría corresponde a reportes de caso. ${ }^{1-14}$ Se presenta el caso de una paciente con un LMS primario óseo en fémur distal izquierdo primario, tratado quirúrgicamente y con quimioterapia, en quien posteriormente, durante el seguimiento, se identificó una lesión metastásica en clavícula con fractura patológica manejada con resección quirúrgica.

\section{Descripción del Caso}

Paciente femenina de 28 años de edad, quien consultó por un cuadro clínico de 2 meses y medio de evolución consistente en dolor en la rodilla derecha, de predominio nocturno y con el apoyo de la extremidad asociado a limitación funcional. Al examen físico, se encontró dolor leve con el apoyo de la extremidad y con la movilización de la rodilla, sin otros hallazgos de importancia, y no había limitación en la movilidad de la rodilla.

Se realizó radiografía de la rodilla derecha, que mostró una lesión lítica en la metafísis distal del fémur, sin compromiso de las corticales. Las imágenes de la resonancia nuclear magnética (RNM) y de la tomografía axial computarizada (TAC) de la rodilla eran compatibles con tumor de células gigantes por lesión lítica en fémur distal, con posible diagnóstico diferencial de osteosarcoma telangiectásico. La gammagrafía ósea evidenció una captación marcada en fémur distal, sin otras lesiones óseas (figuras 1-3).

Se realizó la toma de biopsia en fémur distal en sala de cirugía por vía abierta. El reporte de patología informó la presencia de células fusiformes con extremos romos, citoplasma rosado con áreas vacuoladas organizadas en

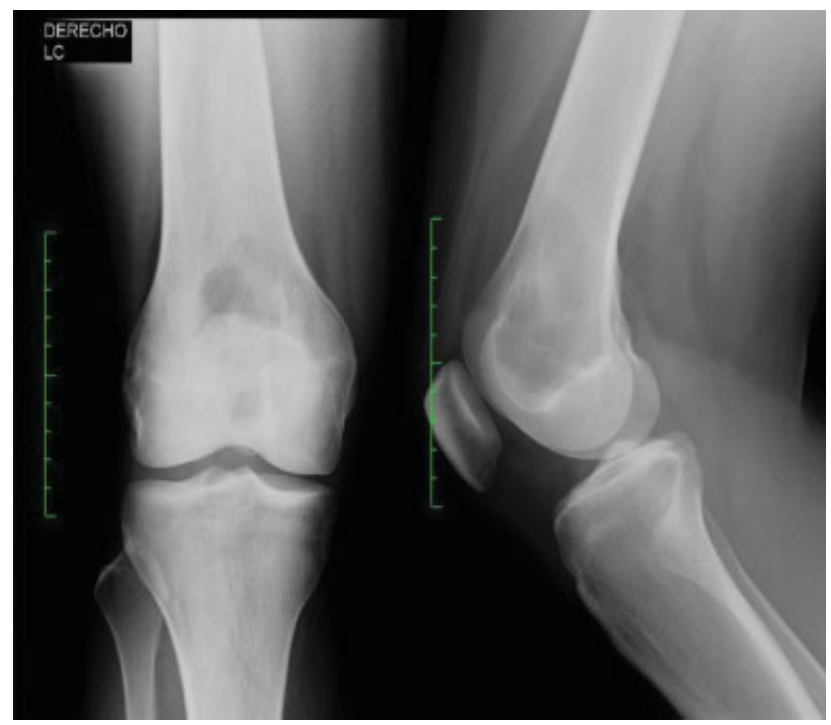

Fig. 1 Radiografías de rodilla anteroposterior y lateral derecha, Que muestra lesión lítica en metáfisis distal del fémur. 


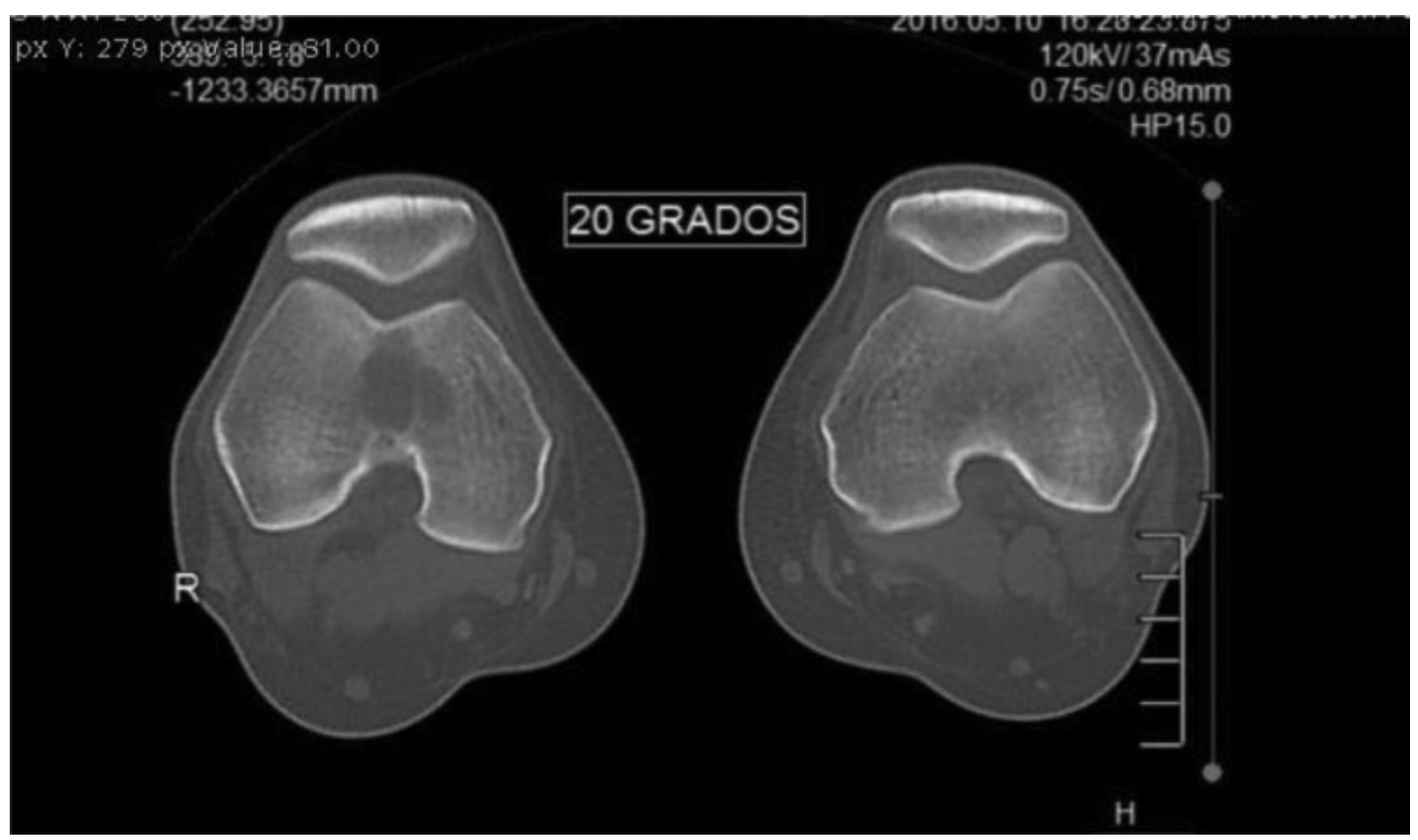

Fig. 2 Tomografía axial computarizada de rodillas comparativa. Nótese la lesión lítica al interior del fémur derecho.

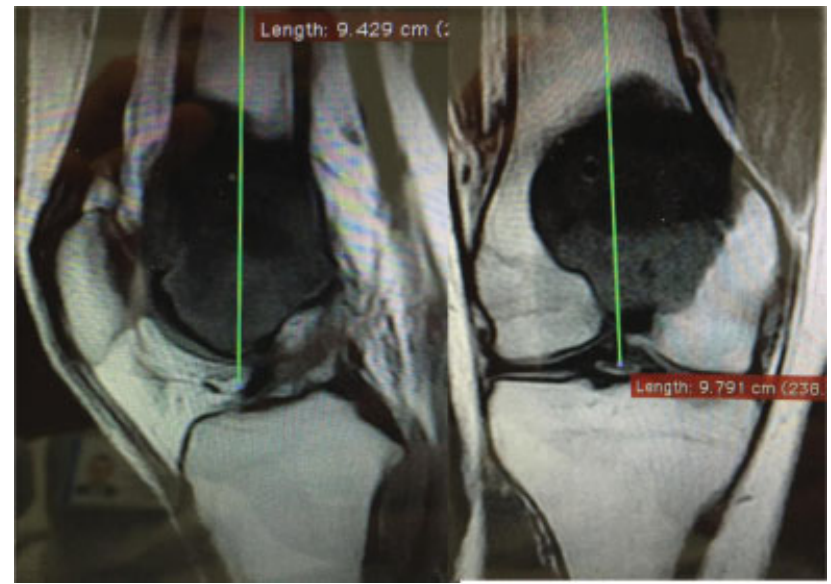

Fig. 3 Resonancia nuclear magnética de rodilla derecha que muestra compromiso extenso de la metáfisis distal del fémur, sin compromiso extraóseo.

fascículos, núcleos tumorales moderadamente pleomórficos, actividad mitótica variable, con un rango de 0 a 5, y áreas de necrosis del $5 \%$. En inmunohistoquímica, había positividad fuerte y difusa para actina muscular, h-caldesmon y miosina. El índice de proliferación Ki67 era de 60\%. No hubo positividad para citoqueratina AE1/Ae3, desmina, proteína S-100, anticuerpo monoclonal HMB458, cúmulo de diferenciación 99 (CD99), ni para receptores estrogénicos, con un diagnóstico de leiomiosarcoma óseo bien diferenciado. Se inició el tratamiento con 3 ciclos de quimioterapia neoadyuvante preoperatoria con ifosfamida $1.000 \mathrm{mg} / \mathrm{m}^{2}$ los días 1 a $3, \mathrm{y}$ doxorrubicina $70 \mathrm{mg} / \mathrm{m}^{2}$.

Se llevó a una segunda cirugía para resección ampliada del tumor y salvamento de la extremidad con endoprótesis de

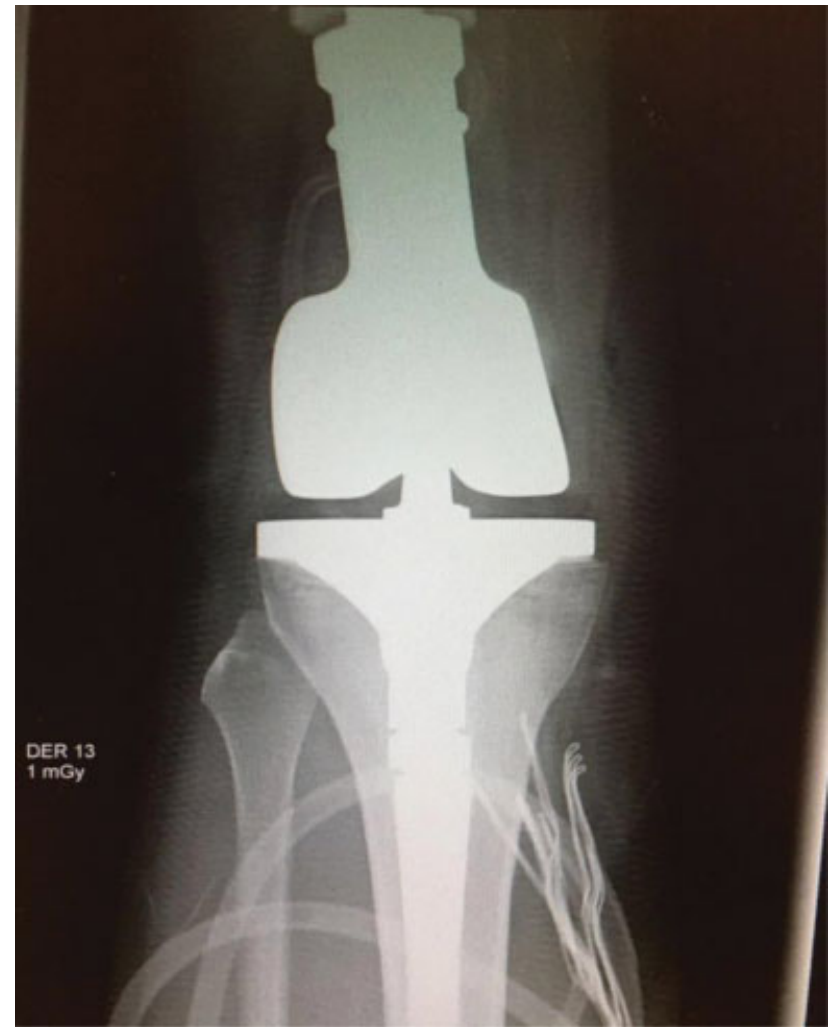

Fig. 4 Radiografía de control postquirúrgico de la rodilla derecha.

rodilla (figura 4), sin complicaciones. La patología confirmó el diagnóstico de un LMS primario de hueso bien diferenciado, con márgenes negativos y $20 \%$ de necrosis (figuras $\mathbf{5}, \mathbf{6}$ ). Posteriormente, la paciente fue sometida a quimioterapia 


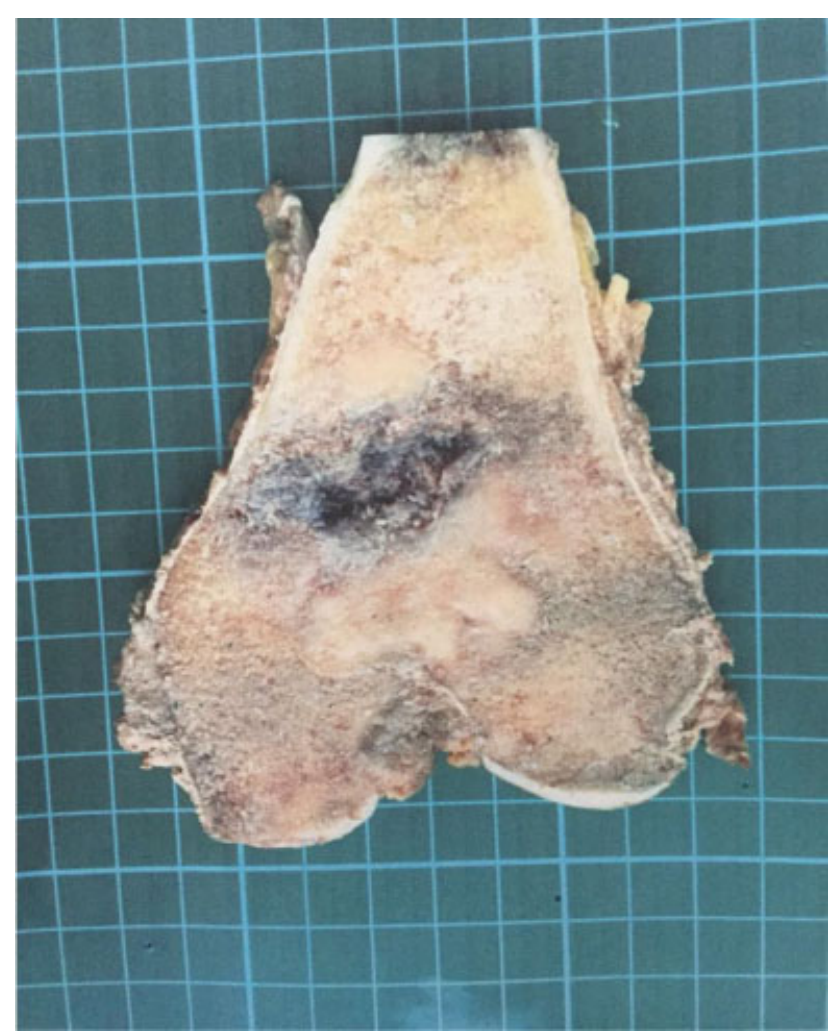

Fig. 5 Espécimen de patología de aspecto macroscópico.

adyuvante, con 4 ciclos de gencitabina $1.000 \mathrm{mg} / \mathrm{m}^{2}$ entre los días 1 y 8, y doxetacel $70 \mathrm{mg} / \mathrm{m}^{2}$ el día 1 .

Durante el seguimiento postquirúrgico, la paciente presentó una adecuada evolución clínica, con una adecuada rehabilitación, con recuperación de la movilidad y funcionalidad de la rodilla derecha. A los dos meses postquirúrgicos, cursó con dolor en la clavícula derecha; se tomó una radiografía, que mostró una fractura en el tercio medio de la clavícula de características patológicas (figura 7), además de una tomografía por emisión de positrones (TEP), que evidenció una lesión a nivel de la clavícula derecha en el tercio medio, compatible con una lesión patológica. Se tomó una biopsia, que reveló una lesión metastásica de LMS óseo, por lo que se realizó resección quirúrgica de la clavícula. Un año y seis meses después de la cirugía, se evaluó la paciente con TEP, sin nueva metástasis (figura 8), sin infección, en buenas condiciones generales, con una adecuada rehabilitación, con radiografías de control sin nuevas lesiones; sin embargo, con reacción osteoblástica en el fémur y la tibia por el uso de prótesis no cementada.

\section{Discusión}

El LMS óseo fue descrito por primera vez en 1965 por Evans y Sanerkin. ${ }^{15}$ Tiene una amplia presentación según edad y género; ${ }^{5-9,14}$ sin embargo, su ocurrencia es muy infrecuente, de menos del $0.7 \%$ de todos los tumores óseos, ${ }^{4,5,7,9}$ y puede tener aparición primaria o metastásica. ${ }^{16}$ En los últimos años, las técnicas de diagnóstico por inmunohistoquímica han mejorado, por lo que esta tasa podría aumentar., ${ }^{32}$ Según la revisión de Adelani et al., ${ }^{17}$ publicada en 2009 con base en 107

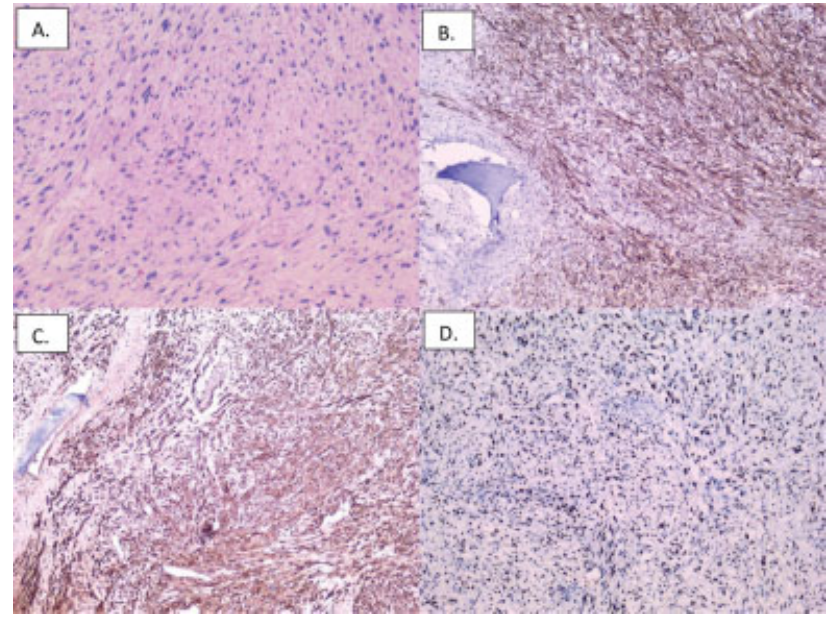

Fig. 6 (A) Células tumorales alargadas con atipia y mitosis. Tinción de hematoxilina y eosina. Aumento de 20x. (B) Tumor positivo (tinción café) para h-caldesmon. Técnica de inmunohistoquímica. Aumento de 20x. (C) Tumor positivo para desmina (tinción café). Técnica especial de inmunohistoquímica. Aumento de 20x. (D) Estudio de índice de proliferación con Ki67 del 30\%. Técnica de inmunohistoquímica. Aumento de 20X. Nota: este marcador indica el porcentaje de células que están en división en un tumor.

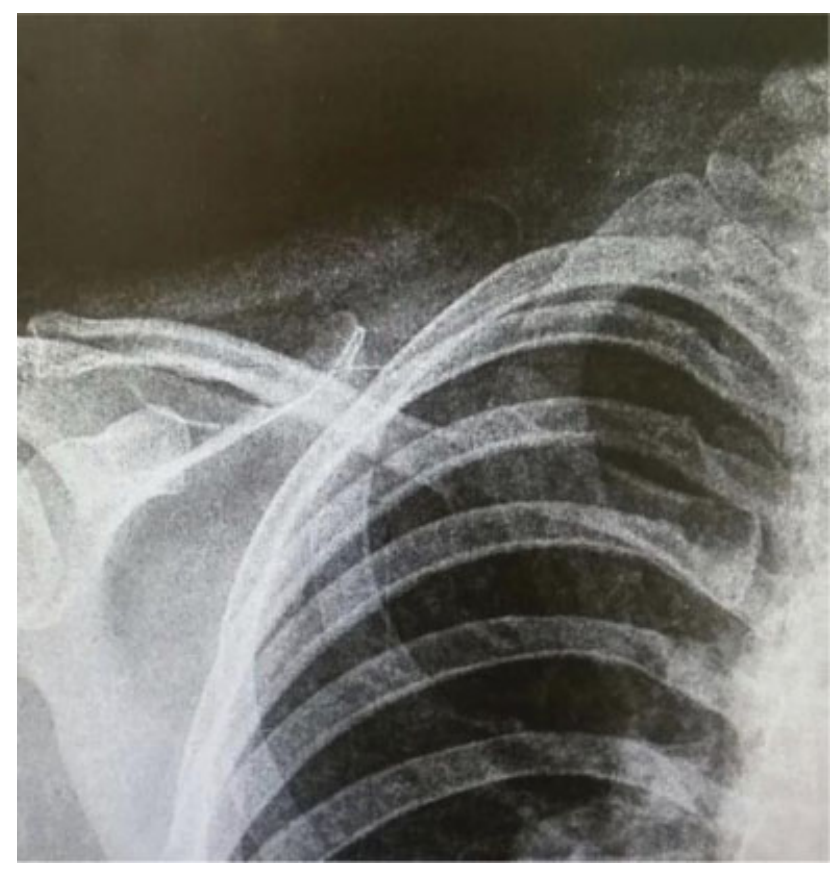

Fig. 7 Radiografía de clavícula derecha con fractura patológica en tercio medio.

casos reportados en la literatura en inglés, se presenta con mayor frecuencia en hombres adultos de 40 años (rango: 9-87 años); en este reporte, el caso corresponde a una paciente de sexo femenino, en edad joven.

Se han planteado varias hipótesis sobre el origen del LMS primario del hueso, probablemente a partir de células vasculares de músculo liso o de fibroblastos capaces de diferenciarse a músculo liso. ${ }^{4,18}$ En algunos reportes, ${ }^{19}$ se ha asociado la infección por el virus de Epstein-Barr (VEB), en el contexto de una inmunosupresión grave (pacientes con 


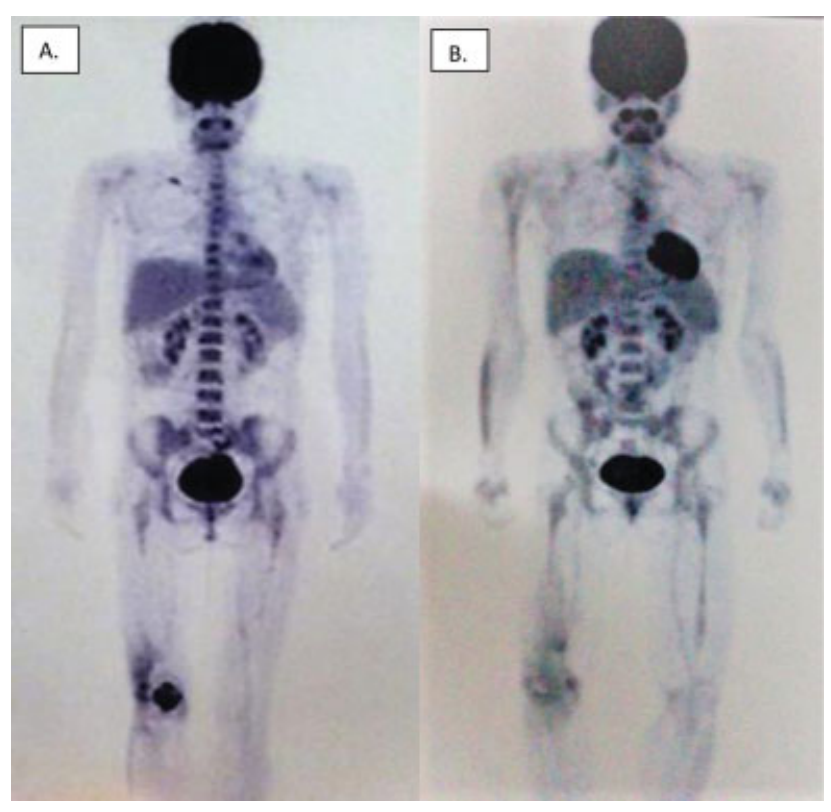

Fig. 8 (A) Tomografía por emisión de positrones que muestra (TEP) lesión metastásica en la clavícula derecha dos meses después de cirugía de salvamento; (B) TEP durante el control a un año y seis meses después de resección de la lesión en el fémur.

síndrome de inmunodeficiencia adquirida [SIDA], y después de trasplantes de riñón, corazón o hígado), con el LMS. Otros factores conocidos para el desarrollo de sarcomas, como la radioterapia, no han demostrado tener relación con el este tipo de tumor. ${ }^{19}$ Se presenta principalmente en hueso largos, como el fémur distal (42-45\% de los casos), como es el contexto del caso descrito aquí, la tibia proximal (25-38\% de los casos), y en el húmero proximal (15\%), predominantemente en la región epifisiometafisaria. Aunque no es muy frecuente, se han reportado casos en los huesos craneofaciales, el radio, la clavícula, el peroné, la pelvis, las falanges, los metatarsianos, y las vertebras. ${ }^{1,5,8-11,14}$ Los síntomas suelen ser inespecíficos y de aparición insidiosa; sin embargo, en algunos estudios ${ }^{5,8,11}$ se ha identificado el dolor como síntoma principal, lo que es concordante con el caso descrito.

Radiológicamente, el LMS de hueso no tiene un aspecto radiográfico patognomónico, y puede ser similar a cualquier otro trastorno primario o secundario. ${ }^{10}$ Se presenta como una lesión osteolítica de comportamiento agresivo, con reacción perióstica, erosión endóstica, un patrón permeativo, bordes mal definidos, con compromiso medular y cortical, y ausencia de esclerosis. ${ }^{7}, 10,11,14$ En el 15\% al 20\% de los casos, se presenta con fractura patológica. ${ }^{4,14}$ En el $20 \%$ de los casos, se identifican áreas de calcificación que generan un desafío en la diferenciación con un osteosarcoma. ${ }^{9}$ En la RMN, se observa una baja intensidad de señal en T1, similar a la del músculo, y una intensidad de señal alta en T2, en general con áreas de compromiso mayor respecto a las radiografías simples, $5,7,16$ que, no obstante, permiten identificar claramente la interfaz entre el tumor y los tejidos circundantes, lo que contribuye a la evaluación correcta de las estructuras adyacentes. ${ }^{14}$ Debe establecerse un diagnóstico diferencial con metástasis de un LMS en tracto gastrointestinal o tejido blando, osteosarcoma intramedular de bajo grado, fibrosarcoma del hueso, condrosarcoma desdiferenciado, metástasis de un carcinoma de células fusiformes, hemangiopericitoma maligno, angiosarcoma epitelioide, hemangioendotelioma epitelioide, $\mathrm{y}$ osteomielitis. ${ }^{1,5,7,8,11}$

El sitio más frecuente de metástasis es el pulmón, seguido de la columna lumbar y el hígado. ${ }^{1,16}$ Otros sitios frecuentes de metástasis incluyen las glándulas suprarrenales, el riñón, el mediastino, los ganglios linfáticos inguinales, y los ganglios linfáticos supraclaviculares; ${ }^{14}$ en el reporte actual, se presentó una metástasis ósea a distancia, localizada en la clavícula derecha.

Macroscópicamente, el LMS óseo aparece como una lesión carnosa de color blanco grisáceo, con áreas de necrosis. ${ }^{18}$ Histológicamente, se caracteriza por la ausencia de matriz condral u osteoide, con infiltración del hueso trabecular, áreas de necrosis o hemorragia, presencia de células fusiformes en general, que se encuentran dispuestas en fascículos desorganizados, con intersecciones en ángulos perpendiculares, atipia celular, pleomorfismo nuclear con núcleos en forma de cigarro, abundante citoplasma eosinofílico y fibrilar, con un perfil inmunohistoquímico para actina, músculo liso y marcadores tumorales como actina, desmina, alfa actina de músculo liso, h-caldesmon, vimentina positivos en tinción. ${ }^{1,2,5,6,9-12}$ En nuestra paciente, se encontró en el estudio histopatológico la presencia de células fusiformes con extremos romos, citoplasma rosado con áreas vacuoladas organizadas en fascículos, núcleos tumorales moderadamente pleomórficos, actividad mitótica variable, con un rango de 0 a 5 , y áreas de necrosis del $5 \%$, con positividad para actina muscular, h-caldesmon, y miosina, lo que corresponde a lo descrito en la literatura. ${ }^{1,2,5,6,9-12}$

La escisión quirúrgica con márgenes amplios se considera el patrón de oro en el manejo de esta patología, pues asegura un tratamiento curativo. Debe reservarse la amputación en casos en que se encuentre comprometido el paquete neurovascular o exista un compromiso extenso en tejidos blandos. ${ }^{1,16}$ El papel de la quimioterapia y radioterapia preoperatoria y postoperatoria sigue siendo discutible; sin embargo, se utilizó como neoadyuvancia en nuestra paciente. ${ }^{1,3,4,6,12}$

Debido a que hay pocos reportes, $2,3,5-8,10-15,17$ es difícil establecer el pronóstico de estas lesiones; no obstante, la mayoría de los estudios reportan un comportamiento agresivo asociado a un pronóstico no poco favorable. Los factores pronósticos que se han relacionado con una menor sobrevida en LMS óseo incluyen: la edad $>40$ años, el tamaño $>8 \mathrm{~cm}$, la presencia de una fractura patológica, la amputación, los márgenes afectados, la presencia de metástasis, el retraso o ausencia de manejo quirúrgico, y una respuesta deficiente a la quimioterapia preoperatoria. ${ }^{3,20} \mathrm{En} \mathrm{su}$ estudio, Brewer et al. ${ }^{16}$ demostraron que el pronóstico se basa en la etapa de diagnóstico; en los casos de estadios 1b o 2a en la clasificación de Enneking, alcanzaron el 100\% de supervivencia, $\mathrm{y}$, en casos de estadio $2 \mathrm{~b}$, tuvieron un $60 \%$ de supervivencia a los 5 años y un $43 \%$ de supervivencia a los 10 y 15 años. ${ }^{16}$ Un estudio $^{3}$ multicéntrico en Japón con 48 pacientes con LMS primario mostró una tasa de supervivencia global a 5 años de $78.3 \%$, y de supervivencia libre de $44.9 \%$; sin embargo, se usó 
quimioterapia neoadyuvante en 18 pacientes sin claridad de su utilidad. La presencia de metástasis en la primera visita y la falta de cirugía definitiva se correlacionaron significativamente con una supervivencia global deficiente, y el margen quirúrgico fue un factor pronóstico significativo para la supervivencia libre de enfermedad. ${ }^{3}$ En una serie de casos, ${ }^{20}$ se evaluó el resultado en 8 pacientes con LMS óseo, se encontró que todos los pacientes desarrollaron metástasis dentro de los 12 meses siguientes del diagnóstico primario, indep-endientemente del grado del tumor; en la paciente descrita aquí, la metástasis se presentó a los 2 meses del procedimiento inicial.

\section{Conclusiones}

Los LMSs primarios del hueso son tumores agresivos, de presentación infrecuente, que deben tratarse de manera agresiva. El manejo quirúrgico radical con márgenes negativos actualmente representa la única opción curativa; el tratamiento coadyuvante con quimioterapia y radioterapia no ha mostrado ser efectivo. Es fundamental en el seguimiento de estos pacientes evaluar la posibilidad de aparición de lesiones metastásicas, de modo que sean tratadas de forma oportuna. Hacen falta más estudios con series de casos a largo plazo, para que puedan establecerse protocolos de tratamiento y pronóstico de este tipo de lesiones.

Financiamiento

La presente investigación no ha recibido ninguna beca específica de agencias de los sectores público, comercial, o sin ánimo de lucro.

Conflicto de Intereses

Los autores no tienen conflicto de intereses que declarar.

\section{Referencias}

1 Hanafy M, Schwonzen M, Kuhnen C, Schley B, Wilke A. Primary leiomyosarcoma of the distal fibula: A case report and review of the literature. Orthop Rev (Pavia) 2018;9(04):7236

2 Bhatnagar N, Lingaiah P, Tiwari A, Mahajan N, Arora S, Dhal A. Primary leiomyosarcoma of femur. J Clin Orthop Trauma 2016;7 (Suppl 1):125-129

3 Mori T, Nakayama R, Endo M, et al. Forty-eight cases of leiomyosarcoma of bone in Japan: A multicenter study from the
Japanese musculoskeletal oncology group. J Surg Oncol 2016;114 (04):495-500

4 Recine F, Bongiovanni A, Casadei R, et al. Primary leiomyosarcoma of the bone: a case report and a review of the literature. Medicine (Baltimore) 2017;96(45):e8545

5 Singh D, Kumar R, Kamau GG, Mmopelwa T, Mhembelo D. Primary leiomyosarcoma of the first metatarsal bone: A case report. SA Orthopaed J 2017;16(01):4-7

6 Goto T, Ishida T, Motoi N, et al. Primary leiomyosarcoma of the femur. J Orthop Sci 2002;7(02):267-273

7 Frings A, Leithner A, Liegl-Atzwanger B. Leiomyosarcoma of bone: a case report. Case Rep Med 2011;2011:980257

8 Chow LTC. Metatarsal leiomyosarcoma masquerading as acute osteomyelitis - A diagnostic trap unveiled by vigilant clinical, radiologic and pathologic analysis. Foot 2016;27:10-15

9 Rekhi B, Kaur A, Puri A, Desai S, Jambhekar NA. Primary leiomyosarcoma of bone-a clinicopathologic study of 8 uncommon cases with immunohistochemical analysis and clinical outcomes. Ann Diagn Pathol 2011;15(03):147-156

10 Murphy SM, Torreggiani WC, Marchinkow LO, et al. Formation médicale continue Radiology for the surgeon. Musculoskelet Care 2003;46(05):361-363

11 Atalar H, Gunay C, Yildiz Y, Saglik Y. Primary leiomyosarcoma of bone: a report on three patients. Clin Imaging 2008;32(04): 321-325

12 Yang Y, Ma L, Li L, Liu H. Primary leiomyosarcoma of the spine: A case report and literature review. Medicine (Baltimore) 2017;96 (09):e6227

13 Ishii T, Sakamoto A, Matsuda S, et al. Leiomyosarcoma in the humerus with leukocytosis and elevation of serum G-CSF. Skeletal Radiol 2012;41(06):719-723

14 Shen SH, Steinbach LS, Wang SF, Chen WY, Chen WM, Chang CY. Primary leiomyosarcoma of bone. Skeletal Radiol 2001;30(10): 600-603

15 Evans DM, Sanerkin NG. Primary leiomyosarcoma of bone. J Pathol Bacteriol 1965;90(01):348-350

16 Brewer P, Sumathi V, Grimer RJ, et al. Primary leiomyosarcoma of bone: analysis of prognosis. Sarcoma 2012;2012:636849

17 Adelani MA, Schultenover SJ, Holt GE, Cates JMM. Primary leiomyosarcoma of extragnathic bone: clinicopathologic features and reevaluation of prognosis. Arch Pathol Lab Med 2009;133(09):1448-1456

18 Overgaard J, Frederiksen P, Helmig O, Jensen OM. Primary leiomyosarcoma of bone. Cancer 1977;39(04):1664-1671

19 Serrano C, George S. Leiomyosarcoma. Hematol Oncol Clin North Am 2013;27(05):957-974

20 Pakos EE, Grimer RJ, Peake D, et al. The 'other' bone sarcomas: prognostic factors and outcomes of spindle cell sarcomas of bone. J Bone Joint Surg Br 2011;93(09):1271-1278 